“ (C) 2013 IEEE. Personal use of this material is permitted. Permission from IEEE must be obtained for all other uses, in any current or future media, including

reprinting/republishing this material for advertising or promotional purposes, creating new collective works, for resale or redistribution to servers or lists, or reuse of any copyrighted component of this work in other works." 


\title{
Effects of Hyperglycemia on Variability of RR, QT and corrected QT intervals in Type 1 Diabetic Patients
}

\author{
LinhLan Nguyen, Steven Su, Member, IEEE, Hung T. Nguyen, SeniorMember, IEEE
}

\begin{abstract}
In this study, we evaluated the effects of hyperglycemia on the variability of RR (HRV), QT interval variability (QTV) and corrected $Q T$ interval variability $(Q T c V)$ during hyperglycemic and non-hyperglycemic conditions in six Type 1 diabetic patients at nights. The aim of this study was to investigate the association of high blood glucose levels with autonomic modulation of heart rate and variation in ventricular repolarization. Blood glucose level (BGL) threshold for defining hyperglycemia state was set at $8.33 \mathrm{mmol} / \mathrm{l}$. Variability of RR, QT and corrected QT intervals during hyperglycemic and non-hyperglycemic were quantified using time and frequency domain measures. Hypomon ${ }^{\circledR}$ device was used to monitor ECG signals and acquire RR and QT intervals in Type 1 diabetic patients overnight. The results indicated that time and frequency domain HRV variables were significantly decreased under hyperglycemic condition and inversely correlated with BGL. QTV parameters also reduced when BGL increased and time domain measures of QTV were inversely associated with BGL. Variability in QTe interval was much less than in the QT interval and demonstrated a lower SDNN and LF power. We concluded that certain components of $H R V$, time-domain measures of QTV and QTe but not QTeV are strongly correlated to high blood glucose levels and can be good markers to identify hyperglycemic events in T1DM.
\end{abstract}

\section{INTRODUCTION}

Variability of RR as known as Heart rate variability (HRV) measures has a close relationship to sympathetic and parasympathetic branches of the autonomic nervous system (ANS) [1]. These branches are linked to the heart at the sinoatrial node (SA) where the beat is initiated for a normal heart. The sympathetic branch will raise heart rate, blood sugar and blood pressure under stress while the parasympathetic branch will have an opposite effect once the body gets rest and relaxation [2]. The use of HRV has emerged as a simple, reliable and non-invasive method to evaluate the sympatho-vagal balance at the sinoatrial level. In addition, diabetes mellitus is one of the main causes of autonomic neuropathy, which can lead to abnormalities in heart rate control [3]. Therefore, it is no doubt that there is association between HRV changes and diabetic patients.

Abnormalities of autonomic activity reflected by reduced HRV are strongly associated with increased risk for cardiac events and mortality $[4,5]$. HRV is influenced by a variety of physiological stimuli and all attention has been focused on various ways of analyzing the variation of $R R$ interval or

Linh Lan Nguyen, Steven Su, and Hung T. Nguyen are with the Centre for Health Technologies, Faculty of Engineering and Information

Technology, University of Technology, Sydney, Broadway, NSW 2007, Australia, (e-mail: Linh.L.Nguyen@ @ student.uts.edu.au;

Steven.Su@uts.edu.au; Hung.Nguyen@utse.edu.au ) heart rate. However, it is known that specific branches of the autonomic nervous system may influence difference parts of the cardiac cycle to differing degrees. For example, the cardiac cycle length (the RR interval) and repolarization (QT interval) are independently controlled by the autonomic system [6].

QT interval variability (QTV) reflects temporal fluctuations in duration of ventricular repolarization. The origins of QTV, particularly the influences of autonomic nervous system activity, remain incompletely understood [7]. Nevertheless, QTV is considered to provide a non-invasive measure of susceptibility to ventricular arrhythmias [8].

The variation analysis can be accomplished in the time or frequency domain. The time domain analysis, the simpler way, calculates a number of variables that describe beat to beat changes. The frequency domain components of HRV signal, derived from its power spectrum analysis, has been commonly used to quantify the relative dominance of parasympathetic and sympathetic influence on the heart [9]. These measures are sensitive indicators of autonomic function in diabetic patients [1] and may provide important insight into the pathogenesis of autonomic neuropathy in hyperglycemia [10]. Typically, frequency domain has been divided into three major groups: high, low and very low frequency bands. High frequency $(0.15-0.4 \mathrm{~Hz})$ is thought to represent the parasympathetic component of the autonomic nervous system. Low frequency $(0.04-0.15 \mathrm{~Hz})$ is mediated by both parasympathetic and sympathetic nervous system. Very low frequency $(0.0033-0.04 \mathrm{~Hz})$ is influenced by many factors, including thermoregulation and the renninangiotensin system [11].

Recently, reduced HRV has been seen among patients with Type 1 diabetic mellitus [12,13] and the main driver to this abnormality appears to be hyperglycemia. Hyperglycemia has also been reported with significant decrease in QT interval and corrected QT interval (by Bazett's formula) $[14,15]$. To our knowledge, there has not been studied previously about the variability of QT interval (QTV) and variability of corrected QT interval (QTcV) related to hyperglycemia. Therefore, we now propose the methodology to study RR, QT and corrected QT interval changes simultaneously in both time and frequency domains under hyperglycemic condition and non-hyperglycemic condition. We also examine the strength of the association of high blood glucose levels with variations in RR interval, QT interval and corrected QT interval. Section II presents an overview of the method used in our study. Results of the study and discussion will be mentioned in section III. Finally, section IV provides the conclusion for this study. 


\section{METHODS}

\section{A. Study}

Six patients with T1DM have been studied for overnight glycemia monitoring at the Princes Margaret Hospital for Children in Perth, Australia. Each patient was monitored overnight for the natural occurrence of hypoglycemia and hyperglycemia. Data were collected with approval from Women's and Children's Health Service, Department of Health, Government of Western Australia, and with informed consent.

HypoMon® from AIMedics Pty Ltd was used to continuously measure and record electrocardiographic (ECG) signals every minute during night. This medical device also provided required ECG parameters (RR interval, QT interval and corrected QT interval by Bazett's formula). Data from HypoMon ${ }^{\circledR}$ is all clean signals to make sure artifacts and ectopic beats have been eliminated. Actual blood glucose levels (BGLs) were collected as reference using Yellow Spring Instruments. In our study, the BGL threshold to define hyperglycemia is set at $8.33 \mathrm{mmol} / \mathrm{l}$. BGL was sampled every 30-minute duration from $9 \mathrm{pm}$ to 6 am corresponding to measured ECG parameters.

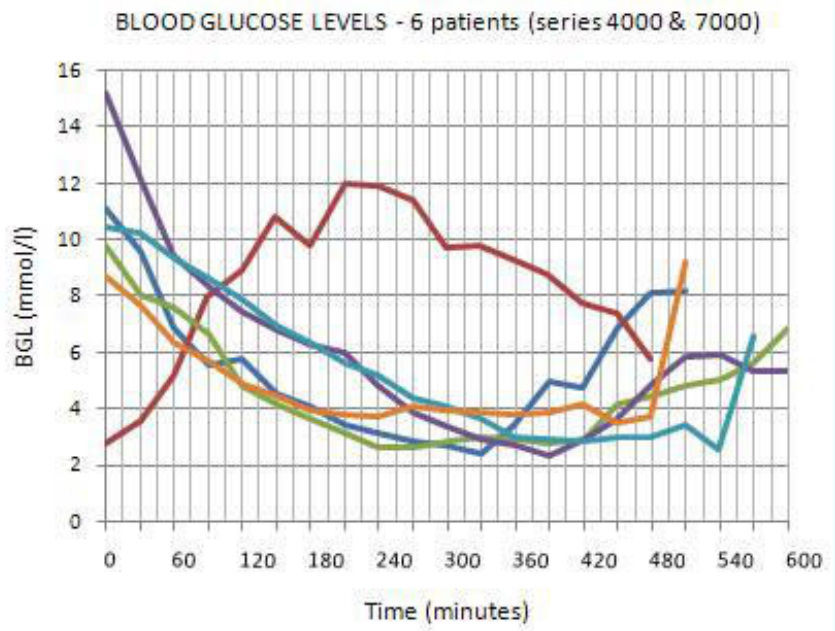

Fig.1.Overnight measurement of Blood glucose level profiles of six T1DM patients

\section{B. Variability assessment}

RR, QT and QTc intervals were analyzed off-line using purpose-written software written in Matlab to calculate the time-domain, frequency domain parameters from raw RR/QT/QTc signals. Baseline drift in data were subtracted by a data-smoothing algorithm. In time domain, we measured mean RR interval, the standard deviation of the RR interval index (SDNN), the root mean square of successive RR interval differences (RMSSD) and the percentage of beats with a consecutive RR interval $>50 \mathrm{~ms}$. SDNN is considered to reflect both sympathetic and the parasympathetic influence on HRV [5]. The other parameters are considered to reliable indices of cardiac parasympathetic activity [16]. Frequency domain analysis was done by fast Fourier transform using Hanning window to derive spectral power. The intervals were linearly interpolated at the rate of $5 \mathrm{~Hz}$ to obtain evenly sampled values. The power of LF, HF and VLF components is usually expressed in absolute units $\left(\mathrm{ms}^{2}\right)$. The ratio between LF and HF components (LF/HF ratio) is used to reflect the balance between sympathetic and parasympathetic parts of the autonomic system and hence shows the level of sympathetic dominance (stress) in a subject [17]. These time and frequency domains parameters are considered to be most physiologically linked to the autonomic nervous system.

\section{Statistical analysis}

Independent $t$-test was applied to every parameter to estimate the significant differences between hyperglycemia and non-hyperglycemia conditions. Moreover, Pearson's correlation analyses were used to evaluate and test the strengths of association between BGLs and variables in time and frequency domains. All statistical analyses were conducted with IBM SPSS version 19 (SPSS Inc, Chicago, IL, USA). Significance value ( $p$-value) less than 0.05 is considered to be significant. Results are presented as mean \pm standard errors.

\section{RESULTS AND DISCUSSION}

\section{A. Heart Rate Variability}

Six out of eight time and frequency domain parameters were significant decreased under hyperglycemic condition compared to non-hyperglycemic condition (as shown in Table I). Mean RR and LF/HF ratio were also reduced but did not achieve statistical significance. This result concurs with other reports that described lower HRV measures in diabetic patients than in normal subjects $[3,12,17]$. Table II demonstrated that BGLs were inversely and significantly correlated with SDNN, RMSSD, pNN50 and LF and HF $(p<0.05)$. It reveals that SDNN appears to be the most significant parameter in HRV with a strong relation with high BGL.

TABLE I. HEART RATE VARIABILITY UNDER HYPERGLYCEMIC AND NON-HYPERGLYCEMIC CONDITIONS

\begin{tabular}{|c|c|c|c|}
\hline $\begin{array}{c}\text { HRV } \\
\text { measures }\end{array}$ & Hyper & Non-hyper & $\boldsymbol{p}$ value \\
\hline Mean RR $(\mathrm{ms})$ & $910.968 \pm 83.002$ & $929.856 \pm 115.096$ & 0.565 \\
\hline SDNN (ms) & $45.499 \pm 15.125$ & $74.099 \pm 27.858$ & $<\mathbf{0 . 0 0 0 1}$ \\
\hline RMSSD (ms) & $57.208 \pm 22.503$ & $86.884 \pm 41.922$ & $\mathbf{0 . 0 1 8}$ \\
\hline pNN50 (ms) & $28.629 \pm 16.676$ & $46.085 \pm 17.971$ & $\mathbf{0 . 0 0 3}$ \\
\hline VLF $\left(\mathrm{ms}^{2}\right)$ & $49.055 \pm 59.687$ & $353.037 \pm 430.255$ & $\mathbf{0 . 0 0 1}$ \\
\hline LF $\left(\mathrm{ms}^{2}\right)$ & $320.968 \pm 252.754$ & $1202.476 \pm 1097.324$ & $\mathbf{0 . 0 0 1}$ \\
\hline HF $\left(\mathrm{ms}^{2}\right)$ & $886.154 \pm 1109.992$ & $1618.507 \pm 1669.221$ & $\mathbf{0 . 0 1 2}$ \\
\hline LF/HF & $0.766 \pm 0.719$ & $1.323 \pm 1.669$ & 0.328 \\
\hline
\end{tabular}

TABLE II. CORRELATIONS BETWEEN HRV AND BLOOD GLUCOSE LEVELS

\begin{tabular}{|c|c|c|}
\hline Variables & Coefficient $\boldsymbol{r}$ & $\boldsymbol{p}$ value \\
\hline Mean RR & -0.034 & 0.842 \\
\hline SDNN & $\mathbf{- 0 . 4 7 3}$ & $\mathbf{0 . 0 0 4}$ \\
\hline
\end{tabular}




\begin{tabular}{|c|c|c|}
\hline Variables & Coefficient $\boldsymbol{r}$ & $\boldsymbol{p}$ value \\
\hline RMSSD & $\mathbf{- 0 . 3 7 3}$ & $\mathbf{0 . 0 2 5}$ \\
\hline pNN50 & $\mathbf{- 0 . 5 2 2}$ & $\mathbf{0 . 0 0 1}$ \\
\hline VLF & -0.322 & 0.056 \\
\hline LF & $\mathbf{- 0 . 3 7 9}$ & $\mathbf{0 . 0 2 3}$ \\
\hline HF & $\mathbf{- 0 . 3 7 2}$ & $\mathbf{0 . 0 2 5}$ \\
\hline LF/HF & -0.199 & 0.246 \\
\hline
\end{tabular}

\section{B. QT Variability}

Summary data on QTV were tabulated in Table III and IV. Except LF/HF ratio, all other parameters in time and frequency domains were significantly lower in hyper events than in non-hyper events $(p<0.05)$. However, when correlation analysis was used, only time domain parameters (Mean QT, SDNN-QT, RMSSD-QT) showed significant relation with high BGLs $(r=-0.429, p=0.004 ; r=-0.429, p$ $=0.004 ; r=-0.429, p=0.004$; respectively) but not frequency domain parameters $(p>0.05)$.

TABLE III. QT VARIABILITY UNDER HYPERGLYCEMIC AND NONHYPERGLYCEMIC CONDITIONS

\begin{tabular}{|c|c|c|c|}
\hline $\begin{array}{c}\text { QTV } \\
\text { measures }\end{array}$ & Hyper & Non-hyper & $\boldsymbol{p}$ value \\
\hline Mean QT (ms) & $379.253 \pm 10.753$ & $392.58 \pm 19.86$ & $\mathbf{0 . 0 2}$ \\
\hline SDNN-QT (ms) & $8.143 \pm 2.728$ & $12.333 \pm 5.749$ & $\mathbf{0 . 0 0 2}$ \\
\hline $\begin{array}{c}\text { RMSSD-QT } \\
(\mathrm{ms})\end{array}$ & $9.78 \pm 3.615$ & $15.584 \pm 7.7$ & $\mathbf{0 . 0 0 1}$ \\
\hline VLF-QT $\left(\mathrm{ms}^{2}\right)$ & $0.877 \pm 1.015$ & $2.457 \pm 3.601$ & $\mathbf{0 . 0 3 4}$ \\
\hline LF-QT $\left(\mathrm{ms}^{2}\right)$ & $3.601 \pm 3.375$ & $11.659 \pm 18.118$ & $\mathbf{0 . 0 2 7}$ \\
\hline HF-QT $\left(\mathrm{ms}^{2}\right)$ & $9.419 \pm 7.795$ & $33.706 \pm 47.488$ & $\mathbf{0 . 0 1 2}$ \\
\hline LF/HF-QT & $0.395 \pm 0.189$ & $0.831 \pm 2.48$ & 0.503 \\
\hline
\end{tabular}

TABLE IV. CORRELATIONS BetweEn QTV AND Blood Glucose LEVELS

\begin{tabular}{|c|c|c|}
\hline Variables & Coefficient $\boldsymbol{r}$ & $\boldsymbol{p}$ value \\
\hline Mean QT & $\mathbf{- 0 . 4 2 9}$ & $\mathbf{0 . 0 0 4}$ \\
\hline SDNN-QT & $\mathbf{- 0 . 3 1 5}$ & $\mathbf{0 . 0 3 7}$ \\
\hline RMSSD-QT & $\mathbf{- 0 . 3 5 1}$ & $\mathbf{0 . 0 2}$ \\
\hline VLF-QT & -0.157 & 0.308 \\
\hline LF-QT & -0.158 & 0.305 \\
\hline HF-QT & -0.166 & 0.282 \\
\hline LF/HF-QT & -0.014 & 0.93 \\
\hline
\end{tabular}

\section{QTc Variability}

QTcV measures were described below in Table V and VI. It can be seen that time domain features were significantly shorter under hyper condition than under non-hyper condition. In frequency domain, only HF component was significantly decreased $(67.35 \pm 65.408$ vs. $244.452 \pm$ $343.639, p=0.019)$. VLF, LF and LF/HF ratio tend to be smaller in hyper compared to non-hyper, but these differences were not statistically significant $(p>0.05)$. In addition, mean QTc seems to be the most related variable to high BGL $(r=-0.497, p=0.001)$ while RMSSD-QT had slight association with high BGL $(r=-0.32, p=0.041)$. Other parameters did not show any statistical correlations with BGL.

TABLE V. CORRECTED QT VARIABILITY UNDER HYPERGLYCEMIC AND NON-HYPERGLYCEMIC CONDITIONS

\begin{tabular}{|c|c|c|c|}
\hline $\begin{array}{c}\text { QTcV } \\
\text { measures }\end{array}$ & Hyper & Non-hyper & $\boldsymbol{p}$ value \\
\hline Mean QTc $(\mathrm{ms})$ & $395.818 \pm 26.789$ & $423.392 \pm 27.053$ & $\mathbf{0 . 0 0 3}$ \\
\hline $\begin{array}{c}\text { SDNN-QTc } \\
(\mathrm{ms})\end{array}$ & $18.12 \pm 6.694$ & $28.922 \pm 19.405$ & $\mathbf{0 . 0 3 9}$ \\
\hline $\begin{array}{c}\text { RMSSD-QTc } \\
(\mathrm{ms})\end{array}$ & $21.625 \pm 8.874$ & $33.445 \pm 17.732$ & $\mathbf{0 . 0 1 8}$ \\
\hline VLF-QTc $\left(\mathrm{ms}^{2}\right)$ & $3.779 \pm 4.558$ & $52.866 \pm 155.678$ & 0.217 \\
\hline LF-QTc $\left(\mathrm{ms}^{2}\right)$ & $19.47 \pm 16.614$ & $245.968 \pm 773.99$ & 0.251 \\
\hline HF-QTc $\left(\mathrm{ms}^{2}\right)$ & $67.35 \pm 65.408$ & $244.452 \pm 343.639$ & $\mathbf{0 . 0 1 9}$ \\
\hline LF/HF-QTc & $0.427 \pm 0.321$ & $0.599 \pm 0.634$ & 0.322 \\
\hline
\end{tabular}

TABLE VI. CORRELATIONS BETWEEN QTCV AND BLOOD GLUCOSE LEVELS

\begin{tabular}{|c|c|c|}
\hline Variables & Coefficient $\boldsymbol{r}$ & $\boldsymbol{p}$ value \\
\hline Mean-QTc & $\mathbf{- 0 . 4 9 7}$ & $\mathbf{0 . 0 0 1}$ \\
\hline SDNN-QTc & -0.226 & 0.155 \\
\hline RMSSD-QTc & $\mathbf{- 0 . 3 2}$ & $\mathbf{0 . 0 4 1}$ \\
\hline VLF-QTc & -0.083 & 0.604 \\
\hline LF-QTc & -0.068 & 0.672 \\
\hline HF-QTc & -0.157 & 0.326 \\
\hline LF/HF-QTc & -0.07 & 0.663 \\
\hline
\end{tabular}

\section{Discussion}

Recent studies have emphasized the importance of HRV in evaluation of autonomic nervous system on the heart rate. Our study demonstrated that it is possible to use time domain measures and power spectral techniques to analyze autonomic influence on the cardiac cycle length and ventricular repolarization. In agreement with previous studies, high blood glucose levels caused lower HRV in subjects with diabetes. Our findings extended the previous observations made in selected T1DM patients and went across the spectrum of hyperglycemia and nonhyperglycemia events. SDNN was much lower in hyperglycemic events than in non-hyperglycemic events means that both the sympathetic and parasympathetic influence on HRV. SDNN is probably the best known HRV 
index [5], mostly used as a time domain measure of HRV, because the additional information from other time domain parameters of HRV is relatively scant. In frequency domain measurements, LF can be used to quantify the sympathetic component of autonomic function in addition to $\mathrm{HF}$, the parasympathetic component. Our results showed that both these two features were significantly decreased under hyperglycemic condition. Therefore, SDNN, LF and HF could be good markers to identify hyperglycemic and nonhyperglycemic states using HRV in T1DM.

There is little known of QTV in both time and frequency domain measurements among diabetes population. Recently, variability of QT interval is also considered to be able to reflect the variation of ventricular repolarization under the influence of the autonomic system in patients with ischemic heart disease [18]. Data from our study confirm these findings also true in diabetes patients by demonstrating the reduction in almost QTV measures. Since the QTc interval was derived to be independent of heart rate, the observed variation of QTc, at least partially, represent a direct modulation of ventricular repolarization by the autonomic system. The QTc variability in frequency domain has not been investigated in diabetes patients before. Clearly, QTV and QTcV are significantly smaller than HRV. QTcV seems to reflect parasympathetic function in the context of high blood glucose due to only HF component showed significant difference. We also explored the relations between variables in QTV and QTcV with BGL. Unlike HRV, only time domain measures of QTV and duration of QTc interval are related to hyperglycemia. It still remains unclear why only time domain measurements show relations with high blood glucose levels, probably because origin of QT variability as well as QTc variability are incompletely understood. Further studies with larger population are essential to draw definite conclusion. Meanwhile, time domain measures of QTV and QTc interval still proved to capable in clarifying states of hyper and non-hyper.

\section{CONCLUSION}

In conclusion, our study demonstrated that variability of RR, QT, and QTc intervals are reduced in patients with T1DM under hyperglycemic condition. We believe this is the first report to identify abnormal QT variability and QTc variability in hyperglycemia. Certain measures of HRV, time domain measures of QTV and QTc but not QTcV have associations with high blood glucose. These variables are feasible input to classify states of hyperglycemia or nonhyperglycemia in T1DM. The prognostic value of these findings remains unknown. Additional clinical studies are needed to determine the mechanisms in the reduction of HRV, QTV and QTcV related to high blood glucose levels. Knowledge of HRV as well as QTV and QTcV may influence the aggressive of intervention and the choice of therapy when treating hyperglycemia and its complications.

\section{REFERENCES}

[1] Task Force of the European Society of Cardiology the North American Society of Pacing Electrophysiology, "Heart Rate Variability," Circulation, vol. 93, pp. 1043-1065, March 1, 1996.

[2] S. J. Shin, W. N. Tapp, S. S. Reisman, and B. H. Natelson, "Assessment of autonomic regulation of heart rate variability by the method of complex demodulation," IEEE Trans Biomed Eng, vol. 36, pp. $274-83,1989$.

[3] H. Kudat, V. Akkaya, A. B. Sozen, S. Salman, S. Demirel, M. Ozcan, D. Atilgan, M. T. Yilmaz, and O. Guven, "Heart rate variability in diabetes patients," J Int Med Res, vol. 34, pp. 291-6, 2006.

[4] H. Tsuji, M. G. Larson, F. J. Venditti, Jr., E. S. Manders, J. C. Evans, C. L. Feldman, and D. Levy, "Impact of reduced heart rate variability on risk for cardiac events. The Framingham Heart Study," Circulation, vol. 94, pp. 2850-5, 1996.

[5] J. T. Bigger, Jr., J. L. Fleiss, R. C. Steinman, L. M. Rolnitzky, R. E. Kleiger, and J. N. Rottman, "Frequency domain measures of heart period variability and mortality after myocardial infarction," Circulation, vol. 85, pp. 164-71, 1992.

[6] J. Forester, H. Bo, J. W. Sleigh, and J. D. Henderson, "Variability of $\mathrm{R}-\mathrm{R}, \mathrm{P}$ wave-to-R wave, and $\mathrm{R}$ wave-to-T wave intervals," $A m J$ Physiol, vol. 273, pp. H2857-60, 1997.

[7] M. Baumert, M. P. Schlaich, E. Nalivaiko, E. Lambert, C. I. Sari, D. M. Kaye, M. D. Elser, P. Sanders, and G. Lambert, "Relation between QT interval variability and cardiac sympathetic activity in hypertension," Am J Physiol Heart Circ Physiol, vol. 300, p. 21, 2011.

[8] Ronald D. Berger, "QT variability," Journal of electrocardiology, vol. 36, pp. 83-87, 2003.

[9] A Malliani, M Pagani, F Lombardi, and S Cerutti, "Cardiovascular neural regulation explored in the frequency domain," Circulation, vol. 84, pp. 482-92, August 1, 1991.

[10] M. Noritake, B. Takase, K. Kudoh, N. Kugai, A. Kurita, and N. Nagata, "Diurnal change in heart rate variability in healthy and diabetic subjects," Intern Med, vol. 31, pp. 453-6, 1992.

[11] H. V. Barron and M. D. Lesh, "Autonomic nervous system and sudden cardiac death," J Am Coll Cardiol, vol. 27, pp. 1053-60, 1996.

[12] Jagmeet P. Singh, Martin G. Larson, Christopher J. O’Donnell, Peter F. Wilson, Hisako Tsuji, Donald M. Lloyd-Jones, and Daniel Levy, "Association of hyperglycemia with reduced heart rate variability (The Framingham Heart Study)," The American journal of cardiology, vol. 86, pp. 309-312, 2000.

[13] M. Jaiswal, E. M. Urbina, R. P. Wadwa, J. W. Talton, R. B. D'Agostino, Jr., R. F. Hamman, T. E. Fingerlin, S. Daniels, S. M. Marcovina, L. M. Dolan, and D. Dabelea, "Reduced Heart Rate Variability Among Youth With Type 1 Diabetes: The SEARCH CVD study," Diabetes Care, vol. 6, p. 6, 2012.

[14] B. Suys, S. Heuten, D. De Wolf, M. Verherstraeten, L. O. de Beeck, D. Matthys, C. Vrints, and R. Rooman, "Glycemia and corrected QT interval prolongation in young type 1 diabetic patients: what is the relation?," Diabetes Care, vol. 29, pp. 427-9, 2006.

[15] Linh Lan Nguyen, Steven Su, and Hung T. Nguyen, "Identification of Hypoglycemia and Hyperglycemia in Type 1 Diabetic patients using ECG parameters," in Engineering in Medicine and Biology Society (EMBC), 2012 Annual International Conference of the IEEE, 2012, pp. 2716-2719.

[16] R. E. Kleiger, P. K. Stein, M. S. Bosner, and J. N. Rottman, "Time domain measurements of heart rate variability," Cardiol Clin, vol. 10, pp. 487-98, 1992.

[17] R. Amanipour, H. Nazeran, I. Reyes, M. Franco, and E. Haltiwanger, "The effects of blood glucose changes on frequency-domain measures of HRV signal in type 1 diabetes," in Electrical Communications and Computers (CONIELECOMP), 2012 22nd International Conference on, 2012, pp. 50-54.

[18] J. S. Sarma, N. Singh, M. P. Schoenbaum, K. Venkataraman, and B. N. Singh, "Circadian and power spectral changes of RR and QT intervals during treatment of patients with angina pectoris with nadolol providing evidence for differential autonomic modulation of heart rate and ventricular repolarization," Am J Cardiol, vol. 74, pp. 131-6, 1994. 